\title{
A Generalized Cole-Hopf Transformation for Nonlinear ODES
}

\author{
Mayer Humi \\ Department of Mathematical Sciences, \\ Worcester Polytechnic Institute, \\ 100 Institute Road, \\ Worcester, MA 01609
}

\begin{abstract}
We introduce a hybrid Cole-Hopf-Darboux transformation to relate solutions of nonlinear and linear second order differential equations and derive a sufficient condition for this correspondence. In particular we show that solutions of some nonlinear second order equations are related to the special functions of mathematical physics through this transformation. These nonlinear equations can be viewed as the "class of special nonlinear equations" which correspond to the linear differential equations which define the special functions of mathematical physics.
\end{abstract}




\section{Background}

In the context of partial differential equations the Hopf-Cole transformation $[1,2]$ and it generalizations $[3,4]$ has been used extensively to linearize some nonlinear partial differential equations such as the Burgers equation [5,6,7].

For ordinary differential equations a "similar" transformation has been used for some time. In fact it is well known [8] that the Ricatti equation

$$
\psi(x)^{\prime}+A(x) \psi(x)^{2}+B(x) \psi(x)+C_{1}(x)=0
$$

where primes denote differentiation with respect to $x$, can be linearized by the transformation

$$
\psi(x)=\frac{\phi(x)^{\prime}}{A(x) \phi(x)} .
$$

The resulting linear equation for $\phi(x)$ is

$$
A(x) \phi(x)^{\prime \prime}+\left(B(x) A(x)-A(x)^{\prime}\right) \phi(x)^{\prime}+C_{1}(x) A(x)^{2} \phi(x)=0
$$

However the operator

$$
T=\frac{d}{d x}+A(x) \psi(x)+B(x)
$$

can be used repeatedly to generate higher order differential equations that can be linearized by the transformation (1.2). That is the differential equation

$$
T^{n} \psi(x)=Q(x)
$$

where $T^{n} \psi(x)=T\left(T^{n-1}\right) \psi(x)$ can be linearized by the transformation (1.2).

In particular for $n=2$ we have

$$
T^{2} \psi=\left(\frac{d}{d x}+A(x) \psi(x)+B(x)\right)\left(\frac{d}{d x}+A(x) \psi(x)+B(x)\right) \psi(x) .
$$

Thus for $n=2$, and $A(x)=1, B(x)=0$ the nonlinear differential equation (1.5) becomes

$$
\psi(x)^{\prime \prime}+3 \psi(x) \psi(x)^{\prime}+\psi(x)^{3}=Q(x) .
$$

Applying the transformation (1.2) to this equation yields

$$
\phi(x)^{\prime \prime \prime}=Q(x) \phi(x)
$$


This shows that the transformation (1.2) relates some nonlinear equations to a linear one of higher order. However there is another class of transformations that are used to relate the solutions of two linear differential equations of the same order. These are Darboux transformations [9-12] (which form the basis for the well known Factorization method [13]). In this case the operator that relates the two equations is of the form

$$
D=C(x)+\frac{d}{d x}
$$

Our objective in this paper is to explore the possible use of some "hybrid" form of (1.2) and (1.9) to relate the solutions of a nonlinear equation to those of a linear one of the same order. In particular we are motivated by the fact that the solutions of some nonlinear second order equations are related to the special functions of mathematical physics through a transformation similar to (1.2). In a certain sense these equations define then a class of "special nonlinear differential equations". Furthermore we show that Painleve II equation has a solution that can be expressed in terms of Airy functions for a special set of its parameters.

The plan of the paper is as follows in Sec. 2 we present the general technique which relates solutions of linear and nonlinear equations. In Sec 3 we specialize to a subset of this general method and provide and intrinsic test for the applicability of the method. Sec 4 explores the relationship between some nonlinear equations and the special functions of mathematical physics. Sec 5 provides some additional examples of nonlinear equations whose solutions are related to those of a linear equation. We end up in Sec. 6 with some conclusions.

\section{A Generalized Transformation}

We shall say that the solutions of the equations

$$
\psi(x)^{\prime \prime}=S(x)+V(x) \psi(x)+W(x) \psi(x)^{2}+R(x) \psi(x)^{3}+\lambda \psi(x)
$$

and

$$
\phi(x)^{\prime \prime}=U(x) \phi(x)+K(x) \phi(x)^{\prime}+\lambda \phi(x)
$$

are related if we can find functions $P(x)$ and $Q(x)$ so that

$$
\psi(x)=P(x)+Q(x) \frac{\phi(x)^{\prime}}{\phi(x)} .
$$


We observe that, in principle, the terms $\lambda \psi(x), \lambda \phi(x)$ in (2.1), (2.2) can be absorbed by $V(x)$ and $U(x)$ respectively. Furthermore (2.1) can take the more general form

$$
\psi(x)^{\prime \prime}=S(x)+V(x) \psi(x)+V_{1}(x) \psi(x)^{\prime}+W(x) \psi(x)^{2}+R(x) \psi(x)^{3} .
$$

In this case we can find $p(x)$ so that $V_{1}(x)=-2 \frac{p(x)^{\prime}}{p(x)}$. Introducing $\xi(x)=p(x) \psi(x)$, (2.4) becomes

$$
\xi(x)^{\prime \prime}=p(x) S(x)+\left(V(x)+\frac{p(x)^{\prime \prime}}{p(x)}\right) \xi(x)+\frac{W(x)}{p(x)} \xi(x)^{2}+\frac{R(x)}{p(x)^{2}} \xi(x)^{3} .
$$

which has the same form as (2.1).

To classify those nonlinear equations (2.1) which can be "paired" with a linear equation of the form (2.2) we differentiate (2.3) twice and in each step replace the second order derivative of $\phi(x)$ by $U(x) \phi(x)+K(x) \phi(x)^{\prime}+\lambda \phi(x)$. We then use (2.1) to eliminate $\psi(x)^{\prime \prime}$. As a result we find that the following equation must hold;

$$
a_{3}(x) \phi(x)^{-3}+a_{2}(x) \phi(x)^{-2}+a_{1}(x) \phi(x)^{-1}+a_{0}(x)=0
$$

where

$$
\begin{gathered}
a_{3}(x)=-Q(x)^{2} R(x)+2, a_{2}(x)=-2 Q(x)^{\prime}-3 R(x) P(x) Q(x)^{2}-W(x) Q(x)^{2}-3 K(x) Q(x) \\
a_{1}(x)=Q(x)^{\prime \prime}+2 K(x) Q(x)^{\prime}- \\
Q(x)\left[3 P(x)^{2} R(x)+2 U(x)+V(x)+2 P(x) W(x)-K(x)^{\prime}-K(x)^{2}+3 \lambda\right] \\
a_{0}(x)=2(U(x)+\lambda) Q(x)^{\prime}+Q(x) U(x)^{\prime}+P(x)^{\prime \prime}-W(x) P(x)^{2}-V(x) P(x)-(2.9) \\
\\
R(x) P(x)^{3}-\lambda P(x)+K(x) Q(x)(U(x)+\lambda)-S(x)
\end{gathered}
$$

To satisfy (2.6) it is sufficient to let $a_{i}(x)=0, i=0,1,2,3$. We use these conditions to express $S(x), V(x), W(x), R(x), K(x)$ and $U(x)$ in terms of the parameters $P(x), Q(x)$. From (2.7) we get

$$
R(x)=\frac{2}{Q(x)^{2}}, \quad W(x)=-\frac{2\left(Q(x)^{\prime}+3 P(x)\right)+3 K(x) Q(x)}{Q(x)^{2}} .
$$


Substituting these results in (2.8) we obtain an equation which we can solve for $V(x)$

$$
\begin{aligned}
V(x)= & \frac{Q(x) Q(x)^{\prime \prime}+4 P(x) Q(x)^{\prime}+6 P(x)^{2}-Q(x)^{2}(2 U(x)-3 \lambda)}{Q(x)^{2}}+ \\
& K(x)^{2}+\frac{\left(6 P(x)+2 Q(x)^{\prime}\right) K(x)}{Q(x)}+K(x)^{\prime} .
\end{aligned}
$$

Substituting these expressions in (2.9) we obtain the following first order linear equation for $U(x)$

$$
\begin{aligned}
& Q(x) U(x)^{\prime}+\left(2 Q(x)^{\prime}+2 P(x)+K(x) Q(x)\right) U(x)+P(x)^{\prime \prime}+ \\
& 2\left\{\lambda-\frac{P(x)}{Q(x)}\left(K(x)+\frac{P(x)}{Q(x)}\right)\right\} Q(x)^{\prime}+2 \lambda P(x)-\frac{P(x)^{2}}{Q(x)^{2}}(2 P(x)+3 K(x) Q(x))- \\
& \frac{P(x) Q(x)^{\prime \prime}}{Q(x)}+K(x)(\lambda Q(x)-K(x) P(x))-P(x) K(x)^{\prime}-S(x)=0 .
\end{aligned}
$$

Equation (2.12) is a linear differential equation for $U(x)$ which can be solved by standard methods once $P(x)$ and $Q(x)$ and $K(x)$ have been specified.

\section{Solutions with $Q(x)=1$}

When $Q(x)=1$ we have $R(x)=2$ and

$$
V(x)=6 P(x)(P(x)+K(x))-2 U(x)-3 \lambda+K(x)^{2}+K(x)^{\prime}, \quad W(x)=-6 P(x)-3 K(x) .
$$

Eq. (2.12) simplifies and we have

$$
\begin{aligned}
& U(x)^{\prime}+2 P(x)\left(U(x)+\lambda-P(x)^{2}\right)+P(x)^{\prime \prime}-S(x)+ \\
& (U(x)+\lambda) K(x)-P(x)\left(K(x)^{2}+3 K(x) P(x)+K(x)^{\prime}\right)=0 .
\end{aligned}
$$

Since this equation contains two unknown functions $U(x)$ and $K(x)$ it is natural to by split it into two equations

$$
K(x)^{\prime}+K(x)^{2}+\left(3 P(x)-\frac{\lambda}{P(x)}\right) K(x)+\frac{S(x)}{P(x)}=0,
$$

and

$$
U(x)^{\prime}+2 P(x)\left(U(x)+\lambda-P(x)^{2}\right)+P(x)^{\prime \prime}+K(x) U(x)=0 .
$$


Eq. (3.3) is independent of $U(x)$ and can be solved for $K(x)$ once $P(x)$ and $S(x)$ have been specified. Actually (3.3) is a Ricatti equation whose linear second order form is

$$
y(x)^{\prime \prime}+\left(3 P(x)-\frac{\lambda}{P(x)}\right) y(x)^{\prime}+\frac{S(x) y(x)}{P(x)}=0
$$

where $K(x)=\frac{y(x)^{\prime}}{y(x}$. We see that by proper choice of $P(x)$ and $S(x)$ the solution $y(x)$ can be a special functions of mathematical physics. In this case $K(x)$ will be the logarithmic derivative of such functions.

Another possible decomposition of (3.2) is to as follows

$$
K(x)^{\prime}+K(x)^{2}+\left(3 P(x)-\frac{\lambda}{P(x)}\right) K(x)=0,
$$

and

$$
U(x)^{\prime}+2 P(x)\left(U(x)+\lambda-P(x)^{2}\right)+P(x)^{\prime \prime}+K(x) U(x)-S(x)=0 .
$$

If we then let $S(x)=-2 P(x)^{3}$, (3.7) becomes linear in both $U(x)$ and $P(x)$. As a result one may choose $P(x)$ and solve this equation for $U(x)$ or choose $U(x)$ and solve for $P(x)$.

Another option to find solutions to (3.2) is to cancel the nonlinear terms in $P(x)$ by making the ansatz

$$
S(x)=-3 P(x)^{2} K(x)-2 P(x)^{3}
$$

the equation then becomes

$$
P(x)^{\prime \prime}+\left(2 U(x)+2 \lambda-K(x)^{\prime}-K(x)^{2}\right) P(x)+K(x)(U(x)+\lambda)+U(x)^{\prime}=0 .
$$

which is a linear equation for $P(x)$ once $U(x)$ and $K(x)$ were chosen.

In the following we use all these strategies to to find nonlinear differential equations whose solutions are related to those of a linear differential equation by the transformation (2.3).

We now consider the following practical question: Suppose one is considering a nonlinear differential equation of the form given by (2.1). Under what conditions one can find a linear differential equation (2.2) whose solutions are related to it by $(2.3)$ with $Q(x)=1$.

Theorem: A sufficient condition for the solution of (2.1) to be related to an equation of the form (2.2) by (2.3) with $Q(x)=1$ is that $R(x)=2$ and

$$
S(x)=\frac{1}{2}\left\{-V(x)^{\prime}+\frac{1}{3}\left[-W(x)^{\prime \prime}+\left(V(x)+W(x)^{\prime}+\lambda\right) W(x)\right]-\frac{1}{27} W(x)^{3}\right\}
$$


When this condition is satisfied one can choose $K(x)=0$ and

$$
U(x)=-\frac{1}{2} V(x)+\frac{1}{12} W(x)^{2}-\frac{3}{2} \lambda
$$

We observe that the condition (3.10) is an intrinsic condition on the coefficients of (2.1).

Proof: From (3.1) we have

$$
P(x)=-\frac{1}{6} W(x)-\frac{1}{2} K(x)
$$

Substituting this expression in the formula for $V(x)$ it follows that

$$
V(x)-\frac{1}{6} W(x)^{2}+\frac{1}{2} K(x)^{2}+2 U(x)+3 \lambda-K(x)^{\prime}=0
$$

Solving (3.13) for $\mathrm{U}(\mathrm{x})$ we find that

$$
U(x)=-\frac{1}{2} V(x)+\frac{1}{12} W(x)^{2}-\frac{1}{4} K(x)^{2}-\frac{3}{2} \lambda+\frac{1}{2} K(x)^{\prime}
$$

Finally substituting these expressions for $P(x)$ and $U(x)$ in (3.2) the condition (3.10) follows. If we let $K(x)=0$ in $(\underline{3.12}),(3.13)$ and (3.14) the expression for $U(x)$ reduces to the one given by (3.11).

\section{Relationships to the Special Functions}

In this section we explore the relationship between some nonlinear equations and the function of mathematical physics. The purpose of our treatment is to highlight this new relationship and is not comprehensive. In all cases we let $Q(x)=1$.

\section{Case 1: Exponential and Trigonometric Functions:}

We start by considering the harmonic oscillator equation,

$$
\phi(x)^{\prime \prime}=-\omega^{2} \phi(x), \quad \omega \neq 0
$$

For this equation $U(x)=-\omega^{2}, K(x)=0$, and $\lambda=0$. Hence from (3.9) we have

$$
P(x)^{\prime \prime}-2 \omega^{2} P(x)=0
$$

Therefore

$$
P(x)=C_{1} e^{\sqrt{2} \omega x}+C_{2} e^{-\sqrt{2} \omega x}
$$


Computing $V(x) W(x)$ and $S(x)$ using (3.1) and (3.8) we finally obtain the following differential equation for $\psi(x)$

$$
\psi(x)^{\prime \prime}-\left(6 P(x)^{2}+2 \omega^{2}\right) \psi+6 P(x) \psi(x)^{2}-2 \psi(x)^{3}=2 P(x)^{3}
$$

whose solutions of are related to those of (4.2) by (2.3) .

If we consider

$$
\phi(x)^{\prime \prime}=\omega^{2} \phi(x)=0, \quad \omega \neq 0
$$

then

$$
P(x)=C_{1} \sin (\sqrt{2} \omega x)+C_{2} \cos (\sqrt{2} \omega x)
$$

and the differential equation for $\psi(x)$ becomes

$$
\psi(x)^{\prime \prime}-\left(6 P(x)^{2}-2 \omega^{2}\right) \psi(x)+6 P(x) \psi(x)^{2}-2 \psi(x)^{3}=-2 P(x)^{3}
$$

For the case where $\omega=0$ i.e.

$$
y(x)^{\prime \prime}=0
$$

then

$$
P(x)=C_{1}+C_{2} x
$$

and the equation for $\psi(x)$ is

$$
\psi(x)^{\prime \prime}-6 P(x)^{2} \psi(x)+6 P(x) \psi(x)^{2}-2 \psi(x)^{3}=-2 P(x)^{3}
$$

Similar treatment can be made for the general second order equation with constant coefficients. We omit the details.

\section{Case 2: Legendre polynomials}

The differential equation for the Legendre polynomials is

$$
\phi(x)^{\prime \prime}=\frac{2 x}{1-x^{2}} \phi(x)^{\prime}-\frac{n(n+1)}{1-x^{2}} \phi(x) .
$$

That is $U(x)=-\frac{n(n+1)}{1-x^{2}}, K(x)=\frac{2 x}{1-x^{2}}$ and $\lambda=0$. Substituting this in (3.9) we find that a particular solution for $P(x)$ is

$$
P(x)=-\frac{2 n(n+1) x}{\left(n^{2}+n-2\right)\left(1-x^{2}\right)}, \quad n \neq 1
$$


(The solution for $n=1$ is available but we shall not elaborate on it further). Using (3.1) we have

$$
V(x)=-\frac{48 n(n+1) x^{2}}{(n+2)^{2}(n-1)^{2}\left(1-x^{2}\right)^{2}}+\frac{2 n(n+1)}{1-x^{2}}+\frac{2\left(2 x^{2}+1\right)}{\left(1-x^{2}\right)^{2}} .
$$

From (3.8) and (3.1) we obtain

$$
\begin{gathered}
S(x)=\frac{8 n^{2}(n+1)^{2}(n+3)(n-2) x^{3}}{(n+2)^{3}(n-1)^{3}\left(x^{2}-1\right)^{3}} . \\
W(x)=-6 P(x)-3 K(x) .
\end{gathered}
$$

For these functions $S(x), V(x), W(x)$ (and $R(x)=2$ ) the solutions of (2.1) are related to the solutions of Legendre equation by the transformation (2.3).

\section{Case 3: Bessel Functions}

The differential equation for Bessel functions is

$$
\phi(x)^{\prime \prime}=-\frac{\phi(x)^{\prime}}{x}-\frac{x^{2}-p^{2}}{x^{2}} \phi(x)
$$

That is $U(x)=-\frac{x^{2}-p^{2}}{x^{2}}, K(x)=-\frac{1}{x}$ and $\lambda=0$. Substituting these in (3.9) we find that the general solutions for $P(x)$ with $p=0,1$ respectively are

$$
\begin{gathered}
P_{0}(x)=\frac{C_{1} e^{z}(\sqrt{2}-2 x)+C_{2} e^{-z}(2 x+\sqrt{2})}{x}+\frac{1}{2 x}, \quad z=\sqrt{2} x, \\
P_{1}(x)=C_{1} e^{z}+C_{2} e^{-z}-\frac{z e^{z} \Gamma(0, z)-z e^{-z} \Gamma(0,-z)}{2 x}+\frac{3}{2 x},
\end{gathered}
$$

where

$$
\Gamma(0, z)=\int_{z}^{\infty} t^{-1} e^{-t} d t
$$

If we choose for $n=0$ the special solution $P_{0}(x)=\frac{1}{2 x}$, we find that

$$
V(x)=2+\frac{1}{2 x^{2}}, \quad W(x)=0, \quad S(x)=\frac{1}{2 x^{3}} .
$$

The differential equation for $\psi(x)$ is

$$
\psi(x)^{\prime \prime}=\frac{1}{2 x^{3}}+\left(2+\frac{1}{2 x^{2}}\right) \psi+2 \psi(x)^{3} .
$$

The solutions of this nonlinear equation are related to the Bessel functions of order zero by the transformation (2.3). 


\section{Case 4: Hermite polynomials}

The differential equation for Hermite polynomials is

$$
\phi(x)^{\prime \prime}=2 x \phi(x)^{\prime}-2 n \phi(x) .
$$

Hence $U(x)=-2 n, K(x)=2 x$. Substituting these in (3.9) we find that an explicit particular (and general) solution for $P(x)$ in terms of elementary functions can be obtained for even $n$. For $n=2,4$ these particular solutions are

$$
\begin{gathered}
P_{2}(x)=-\frac{8\left[\pi\left(1 / 4+x^{2}\right) \exp \left(x^{2}\right) \operatorname{erf}(x)+\sqrt{\pi} x\right]}{\sqrt{\pi}}, \\
P_{4}(x)=-\frac{64\left[\pi\left(3 / 16+3 / 2 x^{2}+x^{4}\right) \exp \left(x^{2}\right) \operatorname{erf}(x)+\sqrt{\pi} x\left(1+x^{2}\right)\right]}{3 \sqrt{\pi}},
\end{gathered}
$$

where

$$
\operatorname{erf}(x)=2 \frac{\int_{0}^{x} \exp \left(-t^{2}\right) d t}{\sqrt{\pi}} .
$$

The computation of the functions $S(x), V(x), W(x)$ using (3.1) and (3.8) is straightforward.

\section{Case 5: Laguerre Polynomials:}

The differential equation for the Laguerre polynomials is

$$
\phi(x)^{\prime \prime}=-\frac{1-x}{x} \phi(x)^{\prime}-\frac{n}{x} \phi(x) .
$$

That is $U(x)=-\frac{n}{x}, K(x)=-\frac{1-x}{x}$ and $\lambda=0$. Substituting these in (3.9) we find that a particular solution for $P(x)$ with $n=2$ is

$$
P_{2}(x)=-\Gamma(0,-x) e^{-x} x+e^{x} \Gamma(0, x)\left(\frac{1}{x}+2 x-2\right)-2+\frac{2}{x}
$$

We omit the computation of $V(x), W(x)$ and $S(x)$ using (3.1) and (3.8) which is straightforward.

\section{Case 6: Painleve II Equation}

Painleve II equation is

$$
\psi(x)^{\prime \prime}=2 \psi(x)^{3}+x \psi(x)+a
$$

where $a$ is a constant. In the notation of the previous sections $V(x)=x, W(x)=0$, $R(x)=2, S(x)=a$ and $\lambda=0$. With $Q(x)=1$ (4.20) satisfies the constraint (3.10) when $a=-\frac{1}{2}$. 
With $K(x)=0$, (3.11) and (3.12) yield under the present settings $P(x)=0$ and

$$
U(x)=-\frac{1}{2} V(x)=-\frac{x}{2}
$$

i.e the differential equation for $\phi(x)$ is

$$
\phi(x)^{\prime \prime}=-\frac{x}{2} \phi(x)
$$

whose general solution is

$$
\phi(x)=C_{1} A i\left(-\frac{x}{2^{1 / 3}}\right)+C_{2} B i\left(-\frac{x}{2^{1 / 3}}\right)
$$

where $A i(x), B i(x)$ are the Airy wave functions. This solution is related to the solution of (4.21) by the transformation (2.3) (with $P(x)=0$ ). We observe that other solutions of this equation are possible if we let $K(x) \neq 0$

\section{Some Special Cases}

In this section we present some explicit solutions to the equations which pair the solution of a nonlinear equation with a linear equation using the algorithm which was presented in the previous sections. In all cases we let $Q(x)=1$.

Example 1: In this example we let $K(x)=0$ and $S(x)=0$ and use (3.2). Choosing $P(x)=\frac{b}{x^{n}}$ we obtain for $n=1$

$$
U(x)=C_{1} x^{-2 b}+\frac{b(b+1)}{x^{2}}-\lambda,
$$

and the corresponding expression for $V(x)$ is

$$
V(x)=2 C_{1} x^{-2 b}+\frac{2 b(2 b-1)}{x^{2}}-\lambda .
$$

For $n \neq 1$ we have

$$
U(x)=C_{1} \exp \left(\frac{2 b x^{1-n}}{n-1}\right)+\frac{n b}{x^{1+n}}+\frac{b^{2}}{x^{2 n}}-\lambda,
$$

and

$$
V(x)=6\left(\frac{b}{x}\right)^{2 n}-2 C_{1} \exp \left(\frac{2 b x^{1-n}}{n-1}\right)-\frac{2 b n}{x^{1+n}}-\frac{2 b^{2}}{x^{2 n}}-\lambda .
$$


For $n=1$ the equation for $\phi(x)$ with $C_{1}=0$ is

$$
\phi(x)^{\prime \prime}-\frac{b(b+1)}{x^{2}} \phi(x)=0,
$$

whose general solution is

$$
\phi(x)=D_{1} x^{b+1}+D_{2} x^{-b} .
$$

The corresponding nonlinear differential equation for $\psi(x)$ is

$$
\psi(x)^{\prime \prime}-\frac{2 b(2 b+1)}{x^{2}} \psi(x)+\frac{6 b \psi(x)^{2}}{x}-2 \psi(x)^{3}=0 .
$$

In this case the explicit relationship between $\phi(x)$ and $\psi(x)$ (as postulated in (2.3) ) is

$$
\psi(x)=\frac{b}{x}+\frac{\phi(x)^{\prime}}{\phi(x)} .
$$

It is straightforward to verify that this is actually a solution of (5.5).

Example 2: Let $P(x)=\frac{-2 x}{3}, S(x)=\frac{-4 n x}{3}$ and $\lambda=0$. Eq. (3.5) becomes

$$
y(x)^{\prime \prime}-2 x y(x)^{\prime}+2 n y(x)=0
$$

which is the differential equation for the Hermite polynomials. Hence

$$
K(x)=\frac{H_{n}(x)^{\prime}}{H_{n}(x)}
$$

where $H_{n}(x)$ is Hermite polynomial of order $n$. Substituting these results in (3.4) leads to the following general solutions for $U(x)$

$$
U_{n}(x)=\frac{\left[C_{1}-\int \frac{16}{27} x^{3} H_{n}(x) \exp \left(-\frac{2 x^{2}}{3}\right) d x\right] \exp \left(\frac{2 x^{2}}{3}\right)}{H_{n}(x)} .
$$

The integral in (5.9) can be computed explicitly for different values of $n$. For $H_{0}(x)=1$ we have

$$
U_{0}(x)=C_{1} \exp \left(\frac{2 x^{2}}{3}\right)-\frac{3}{8}\left(3+2 x^{2}\right)
$$

For $H_{2}(x)=4 x^{2}-2$

$$
U_{2}(x)=\frac{C_{1} \exp \left(\frac{2 x^{2}}{3}\right)-\frac{3}{4}\left(4 x^{4}+10 x^{2}+15\right)}{4 x^{2}-2}
$$


etc.

From these expressions for $K(x)$ and $U(x)$ we find that

$$
V_{n}(x)=\frac{8 x^{2}}{3}-2 U_{n}(x)+\frac{H_{n}(x)^{\prime \prime}-4 x H_{n}(x)^{\prime}}{H_{n}(x)}, \quad W_{n}(x)=4 x-\frac{3 H_{n}(x)^{\prime}}{H_{n}(x)}
$$

We conclude that the solutions of the nonlinear equation (2.1) with $R(x)=2$ and $V_{n}(x), W_{n}(x)$ in (5.10) are related to those of the linear differential equation (2.2) with $U(x)$ and $K(x)$ given by (5.9) and (5.8) by the transformation (2.3).

Example 3: In this example we let $K(x)=0, P(x)=a+b x, S(x)=-2 P(x)^{3}$ and use (3.7) to compute $U(x)$. This yields

$$
\left.U(x)=C_{1} \exp [-(2 a+b x) x]-\lambda, \quad V(x)=6(a+b x)^{2}-\lambda-2 C_{1} \exp [-(2 a+b x) x]\right)
$$

and $W(x)=-6 P(x)($ from $(2.10))$.

Example 4: Here as in the previous example we let $K(x)=0, S(x)=-2 P(x)^{3}$ and $\lambda=0$. However we now let $U(x)=-\frac{a}{x^{2}}$ and use (3.7) to compute $P(x)$. For $a \neq 1$ we obtain

$$
P(x)=\frac{a}{(a-1) x}+C_{1} x^{1 / 2+\beta}+C_{2} x^{1 / 2-\beta} \quad \beta=\sqrt{1+8 a} .
$$

For $a=1$

$$
P(x)=\frac{6 \ln x+2}{x}+C_{1} x^{2}+\frac{C_{2}}{x} .
$$

The corresponding $V(x)$ in this case (viz. $a=1$ ) is

$$
V(x)=\frac{2}{x^{2}}+P(x)^{2} .
$$

If we reverse the roles of $P(x)$ and $U(x)$ i.e fix $P(x)=-\frac{a}{x^{2}}$ and use (3.7) to compute $U(x)$ we find

$$
U(x)=C_{1} \exp \left(-\frac{2 a}{x}\right)-3\left(\frac{1}{x^{2}}-\frac{1}{a x}+\frac{1}{2 a^{2}}\right) .
$$

Example 5: Under the same settings of example 9 we specify $U(x)=2 \exp (2 a x)$ and use (3.7) to compute $P(x)$. We find

$$
P(x)=C_{1} J_{0}(z)+C_{2} Y_{0}(z), \quad z=\frac{2 \exp (a x)}{a}
$$

where $J_{0}$ and $Y_{0}$ are Bessel functions of order zero of the first and second kind. The corresponding values of $V(x)$ and $W(x)$ are

$$
V(x)=6 P(x)^{2}-4 e^{2 a x}, \quad W(x)=-6 P(x) .
$$




\section{Conclusions}

We demonstrated in this paper that the hybrid "Cole-Hopf-Darboux" operator (2.3) can be used to relate the solutions of some nonlinear and linear second order differential equations. The algorithm is straightforward to apply and we presented several examples which demonstrated the scope of the method and its potential interest for problems in mathematical physics. From a perspective those nonlinear differential equation whose solutions can be ex-

pressed in terms of the special functions of mathematical physics (through the transformation (2.3) should obviously be viewed as a new "special class of nonlinear equations".

Research into the possible application of the transformation (2.3) to partial differential equations is on going.

\section{References}

1 E. Hopf - The partial differential equation $u_{t}+u u_{x}=u_{x x}$, Commun. Pure Appl. Math 3, pp.201-230 (1950)

2 J.D. Cole, On a quasi-linear parabolic equation occurring in aerodynamics, Quart. Appl. Math. 9, pp.225-236 (1951)

3 P. L. Sachdev-A generalised Cole-Hopf transformation for nonlinear parabolic and hyperbolic equations, ZAMP 29, No 6 (1978), pp. 963-970, DOI: 10.1007/BF01590817

4 B. Mayil Vaganan- Cole-Hopf Transformations for Higher Dimensional Burgers Equations With Variable Coefficients, Studies in Applied Mathematics, DOI: 10.1111/j.14679590.2012.00551.x (in press) (2012)

5 G Kaniadakis and A M Scarfone - Cole-Hopf-like transformation for Schrodinger equations containing complex nonlinearities, J. Phys. A: Math. Gen. 35, p. 1943 (2002).

6 B. Gaffet-On the integration of the self-similar equations and the meaning of the ColeHopf Transformation J. Math. Phys. 27, 2461 (1986) 
7 Wen-Xiu Ma- An exact solution to two-dimensional Korteweg-de Vries-Burgers equation, J. Phys. A: Math. Gen. 26 L17 (1993).

8 E.L. Ince - Ordinary Differential Equations, Dover Publications, New-York, 1956

9 C. Gu, H. Chaohao and Z. Zhou - Darboux Transformations in Integrable Systems, Springer, New-York (2005)

10 M. Humi - Separation of coupled systems of differential equations by Darboux transformation. J. of Physics A 18, p. 1085 (1985).

11 M. Humi - Darboux transformations for Schroedinger equations in two variables, J. Math. Phys. 46, 083515 (2005) (8 pages).

12 M. Humi- Separation of Coupled Systems of Schroedinger equations by Darboux transformation, Reviews in Mathematical Physics Vol. 24, No. 3, 14 pages (2012)

13 L. Infeld and T.E. Hull - The Factorization method, Rev. Mod. Phys. 23, p.21 (1951). 